\title{
The effects of tea on psychophysiological stress responsivity and post-stress recovery: a randomised double-blind trial
}

\author{
Andrew Steptoe • E. Leigh Gibson • Raisa Vuononvirta • \\ Emily D. Williams • Mark Hamer • Jane A. Rycroft • \\ Jorge D. Erusalimsky • Jane Wardle
}

Published online: 10 November 2006

(C) Springer-Verlag 2006

The online version of the original article can be found at http://dx.doi. org/10.1007/s00213-006-0573-2.

A. Steptoe $(\bowtie) \cdot$ E. L. Gibson · R. Vuononvirta • E. D. Williams • M. Hamer J. Wardle

Department of Epidemiology and Public Health,

University College London,

1-19 Torrington Place,

London WC1E 6BT, UK

e-mail: a.steptoe@ucl.ac.uk

J. A. Rycroft

Unilever Research Colworth,

Bedford, UK

J. D. Erusalimsky

Wolfson Institute for Biomedical Research,

University College London,

London WC1E 6BT, UK
Psychopharmacology (2006) DOI 10.1007/s00213-0060573-2

The name of the third author was misspelt. It should read Raisa Vuononvirta. 